\title{
Caracteres vegetativos y reproductivos de Bromelia serra (Bromeliaceae): Variaciones a distintas escalas espaciales y efectos del ambiente lumínico
}

\author{
Eugenio Alvarez Arnesi ${ }^{12,1}$; Graciela N. Klekailo; ${ }^{1}$ Rodrigo Freire ${ }^{12}$; Andrés \\ Cococcioni'; Jorgelina P. Asmus' ${ }^{12} ;$ Ángeles Tessore $^{1}$ \& Ignacio M. BARberis ${ }^{1 / 2}$
}

${ }^{1}$ Facultad de Ciencias Agrarias. Universidad Nacional de Rosario, Zavalla, Santa Fe. ${ }^{2}$ IICAR (Instituto de Investigaciones en Ciencias Agrarias de Rosario). Universidad Nacional de Rosario - Consejo Nacional de Investigaciones Científicas y Técnicas, Zavalla, Santa Fe.

\begin{abstract}
RESUMEN. Las bromeliáceas terrestres son comunes en los bosques neotropicales. En particular, en el sotobosque de los quebrachales chaqueños existen parches densos de Bromelia serra, que crecen bajo condiciones microambientales heterogéneas. El ambiente lumínico afecta la asignación de biomasa a distintos órganos, por lo que las plantas que crecen bajo distintas condiciones lumínicas presentan una arquitectura diferente. A su vez, el tamaño de la planta y los factores ambientales y genéticos condicionan la asignación de biomasa a los caracteres reproductivos. Para evaluar el efecto del ambiente lumínico sobre los caracteres vegetativos y reproductivos se seleccionaron cuatro parches en cada uno de tres sitios separados por $500 \mathrm{~m}$, en un quebrachal de la Cuña Boscosa Santafesina. En cada parche se seleccionaron cuatro plantas adultas. A cada planta se le midió la altura, el diámetro, la longitud y el ancho de la hoja más larga, el diámetro de la infrutescencia, el número, el peso y el tamaño de los frutos y el número de semillas. La caracterización de la apertura del dosel sobre cada planta se hizo con fotografía hemisférica. Los Modelos Lineales Generales Mixtos muestran que el porcentaje de la varianza explicado por la variación espacial (factores aleatorios representando a los sitios y a los parches) es mayor para caracteres vegetativos que para reproductivos. La mayor proporción de esta varianza se registró a nivel de parche para los caracteres vegetativos y a nivel de sitio para los reproductivos. Mediante un Modelo de Ecuaciones Estructurales basado en Modelos Lineales Generales Mixtos se determinó que el ambiente lumínico afecta de forma directa e indirecta el diámetro de la infrutescencia. Las plantas expuestas a mayor apertura de dosel son más pequeñas y tienen menor cantidad y tamaño de frutos. Esto resulta en infrutescencias más chicas, lo cual destaca el efecto indirecto del ambiente lumínico sobre los caracteres reproductivos.
\end{abstract}

[Palabras clave: apertura del dosel, asignación de biomasa, Bromeliaceae, plasticidad fenotípica, quebrachal]

\begin{abstract}
Aвstract. Vegetative and reproductive traits of Bromelia serra (Bromeliaceae): Variations at different spatial scales and light environment effects. Terrestrial bromeliads are common in neotropical forests, particularly in the understory of the Chaco woodlands, where there are dense patches of Bromelia serra growing under heterogeneous micro-environmental conditions. The light environment affects the allocation of biomass to different organs and, thus, plants that grow under different light conditions have different architecture. Furthermore, the allocation of biomass to reproductive structures is conditioned by plant size, as well as by environmental and genetic factors. To evaluate the effect of light environment on the vegetative and reproductive traits, four patches were selected from three sites separated by $500 \mathrm{~m}$ in the Cuña Boscosa Santafesina. At each patch, four plants were selected. Height, diameter, length and width of the longest leaf, the diameter of the infructescence, number of fruits, weight, and size of them were measured for each plant. In addition, a hemispheric photograph was taken to characterize the canopy opening. The General Linear Mixed Models showed that the proportion of variation explained by random factors (site and patches) is higher for vegetative than for reproductive traits. Most variation was recorded at the patch level for vegetative traits and at the site level for reproductive traits. The Structural Equation Modeling based on General Linear Mixed Models showed that the diameter of the infructescence was directly and indirectly affected by the light environment. The indirect effect was mediated by the smaller size of plants exposed to a larger opening canopy and by fewer and smaller fruits that resulted in smaller infructescences. These results highlight the indirect effects of light environment on reproductive traits.
\end{abstract}

[Keywords: biomass allocation, Bromeliaceae, canopy openness, phenotypic plasticity, quebrachal]

Editor asociado: Fernando Milesi
Recibido: 26 de Septiembre de 2018

Aceptado: 12 de Julio de 2019 


\section{INTRODUCCIÓN}

Las especies de plantas difieren ampliamente en la asignación de recursos hacia sus caracteres vegetativos y reproductivos (Waller 1988; Grime and Pierce 2012); incluso, existen diferencias marcadas entre individuos dentro de una población (Harper 1977; Bazzaz 1991; Weiner et al. 2009). Numerosos factores afectan la asignación de recursos; entre los abióticos se destacan la disponibilidad de recursos (i.e., luz, agua y nutrientes) y las condiciones ambientales (i.e., $\mathrm{pH}$ y temperatura), mientras que entre los factores bióticos son importantes la presencia de polinizadores, de herbívoros, de competidores y de patógenos (Hendrix 1988; Lovett Doust and Lovett Doust 1988; Zimmerman 1988; Herrera 2017). Los factores genéticos también pueden tener un gran efecto: muchas especies de plantas cultivadas fueron seleccionadas por el hombre sobre la base de la asignación de recursos para la reproducción (Satorre et al. 2003).

En muchas especies, el tipo de reproducción condiciona la asignación de recursos. La reproducción sexual se considera ventajosa en los ambientes hostiles, mientras que en aquellos ambientes más estables, la reproducción asexual (clonal mediante bulbos, estolones o rizomas) es considerada más exitosa (Liu et al. 2010). La combinación de ambos tipos de reproducción resulta ventajosa en ambientes cambiantes y muy heterogéneos (de Russo Godoy et al. 2018), tal como ocurre en numerosas comunidades leñosas (Keddy 2017). En plantas con elevada plasticidad fenotípica, la heterogeneidad ambiental puede afectar la asignación de recursos a la reproducción tanto de manera directa como indirecta (Bazzaz et al. 2000; Weiner 2004). Los sitios con mayor disponibilidad de recursos o menor stress permiten, en general, un mejor desarrollo de las plantas y, por consiguiente, una mayor asignación de recursos a la reproducción (Bazzaz et al. 2000).

En el sotobosque de los bosques neotropicales es frecuente la presencia de densas colonias de bromeliáceas terrestres que se reproducen tanto de manera sexual como asexual (Lee et al. 1989; Villegas 2001; Barberis et al. 2014). Como respuesta a la exposición a la luz solar, muchas especies de esta familia presentan una plasticidad fenotípica elevada en sus caracteres vegetativos (Lee et al. 1989; Scarano et al. 2002; Cavallero et al. 2009; Voltolini and Santos 2011; González-Salvatierra et al. 2013; Barberis et al.
2017). Sin embargo, existe poca información sobre el grado de plasticidad fenotípica y sobre los factores que la determinan para caracteres reproductivos en bromeliáceas terrestres (Bartholomew et al. 2003; Montero et al. 2017; Freire et al. 2018). Entre aquellas plantas que se reproducen sexualmente, las de mayor tamaño suelen presentar infrutescencias con mayores diámetros y mayor número de frutos (Overbeek 1946; Bartholomew et al. 2003; Fassinou Hotegni et al. 2015; Silva and Varassin 2016; Montero et al. 2017). En plantas cultivadas de ananá (Ananas comosus (L.) Merr.), las infrutescencias más grandes están dadas por un mayor número de frutos de mayor tamaño y, a su vez, el número de frutos condiciona su propio tamaño (Bartholomew et al. 2003). Una gran variedad de animales frugívoros consume los frutos de las bromeliáceas, lo que permite la dispersión de semillas y la colonización más allá de la planta madre(Benzing 2000). La reproducción asexual mediante rizomas o estolones les permite colonizar rápidamente aquellos espacios con las condiciones ambientales (principalmente de suelo) apropiadas para su establecimiento (Benzing 2000). Las plantas de una colonia o parche provienen de unos pocos individuos originados sexualmente (a partir de semillas dispersadas por animales), que mediante reproducción asexual conformarían un grupo de pocos individuos que comparten un mismo acervo genético (Barbará et al. 2008). Si esto fuese así, se espera que exista mayor similitud en los caracteres vegetativos y reproductivos entre plantas de un mismo parche que entre plantas de distintos parches. No conocemos estudios de bromeliáceas terrestres que hayan analizado las variaciones de los caracteres vegetativos y reproductivos a estas escalas espaciales.

En el Chaco Húmedo argentino, el bosque semiabierto de xerófitas dominado por Schinopsis balansae Engl. ('Mosaico de Bosque de xerófitas, de mesófitas, Sabana y Selva de albardón' [Unidad 15] sensu Oyarzabal et al. 2018) se caracteriza por la presencia de parches boscosos en áreas convexas y parches abiertos con vegetación herbácea en áreas bajas (Lewis 1991; Barberis et al. 1998). En el sotobosque de los sectores boscosos es frecuente la presencia de densas colonias de dos bromeliáceas: Aechmea distichantha Lem. y Bromelia serra Griseb. (Barberis et al. 2002, 2014). Las plantas de esta última especie presentan una plasticidad fenotípica alta (Barberis et al. 2017). Los individuos que crecen al sol son de 
menor tamaño (tienen un menor diámetro y hojas más cortas) y presentan infrutescencias más pequeñas que los individuos ubicados a la sombra (Montero et al. 2017). A su vez, el tamaño de la planta de Bromelia serra afectaría el tamaño de la infrutescencia a través del número y el tamaño de los frutos, tal como se mencionara para $A$. comosus (Bartholomew et al. 2003).

Los objetivos de este estudio fueron a) evaluar las variaciones a distintas escalas espaciales de los caracteres vegetativos y reproductivos en individuos de Bromelia serra de un bosque del Chaco Húmedo y b) analizar el efecto de la apertura del dosel sobre los caracteres reproductivos de esta bromeliácea terrestre.

\section{MATERIALES y MÉTODOS}

\section{Sitio de estudio}

El estudio fue realizado en un bosque de 400 ha de S. balansae (i.e., 'quebrachal') (Lewis et al. 1997) en el Centro Operativo Experimental Provincial 'Aldo Emilio Tessio' (292' S - 60²8' O.; 58 m s. n. m.), ubicado en Las Gamas, provincia de Santa Fe, Argentina. El clima es templado húmedo a cálido, con una temperatura media anual de $20^{\circ} \mathrm{C}$. La precipitación media anual es de $1090 \mathrm{~mm}$, con gran variabilidad interanual: entre 529 y $2060 \mathrm{~mm} /$ año (períodos obtenidos entre los períodos 1911-1950 y 1986-2010). Las lluvias son principalmente estivales (diciembremarzo), y durante el invierno se presenta una temporada seca de duración variable. El suelo del quebrachal presenta baja conductividad hidráulica y alto contenido de sodio (Barberis et al. 1998). Aun cuando la pendiente general es muy baja, existe un marcado micro-relieve con diferencias de altura de hasta $50 \mathrm{~cm}$ en distancias de unos pocos metros (Barberis et al. 1998).

Bromelia serra crece formando colonias o parches en el sotobosque del quebrachal (Figura 1a) en sectores relativamente elevados rodeados por una matriz de pastizal en suelos más bajos (Lewis 1991; Barberis et al. 2002; Barberis and Lewis 2005). Se propaga tanto de forma sexual como asexual (Smith and Downs 1979; Bianchi et al. 2000; Klekailo et al. 2012); la reproducción asexual es mediante estolones largos en cuyo extremo se desarrolla un nuevo individuo (Smith and Downs 1979; Arenas and Arroyo 1988). Tal como en otras especies del género Bromelia (Canela and Sazima 2005; de Russo Godoy et al. 2018), sus flores son polinizadas por picaflores e insectos (J. L. Vesprini, observación personal). La infrutescencia tiene forma globosa (Figura 1b) y puede tener docenas de bayas tricarpelares amarillas de $4 \times 2.5 \mathrm{~cm}$ (Nájera 1974; Caffini et al. 1988; Montero et al. 2017), que contienen de cinco a 50 semillas de color marrón-rojizas (5 $\mathrm{mm}$ largo $\times 7 \mathrm{~mm}$ alto $\times 3 \mathrm{~mm}$ de ancho máximo), de forma lenticular o discoidea (Amat 1988). a)

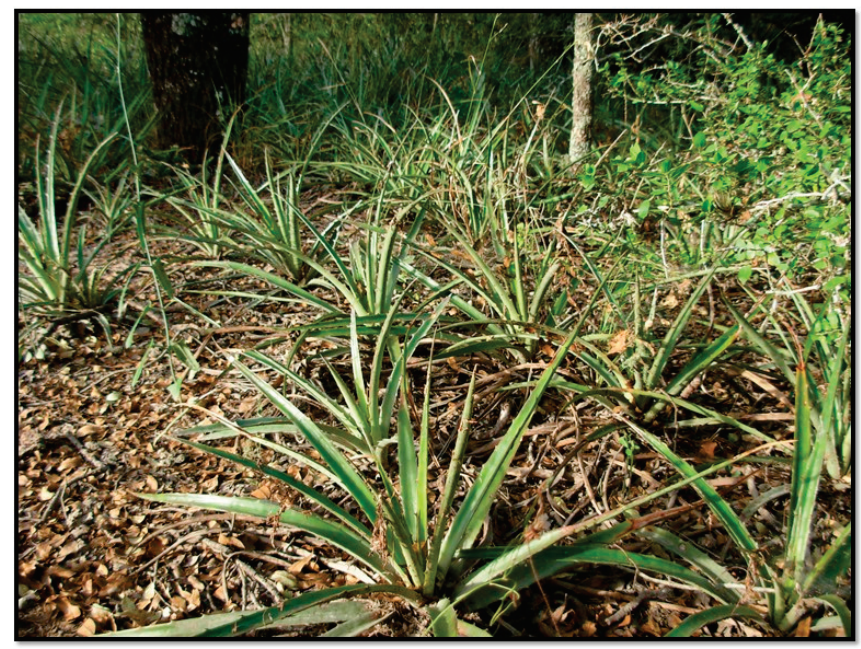

b)

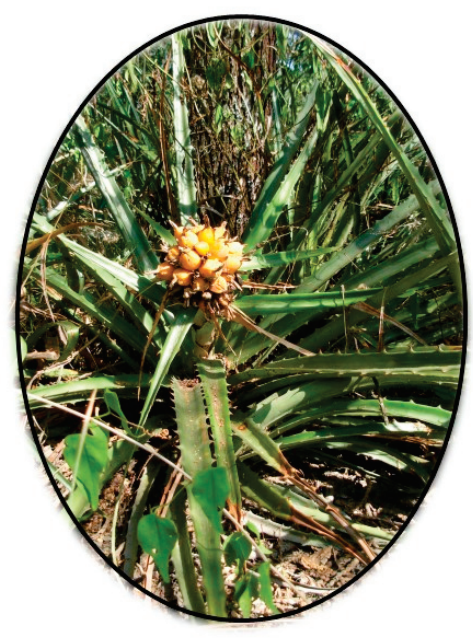

Figura 1. a) Parche de individuos de B. serra en el sotobosque del quebrachal (Las Gamas, Santa Fe, Argentina). b) Planta reproductiva de $B$. serra con infrutescencia de frutos maduros.

Figure 1. a) Bromelia serra patch in the understory of the quebrachal (Las Gamas, Santa Fe, Argentina). b) Reproductive plant of B. serra showing an infructescence with ripe fruits. 
Numerosas especies de invertebrados y de vertebrados consumen estos frutos (Parera 2002; Montero et al. 2017). Al igual que en otras especies del género Bromelia (PaulinoNeto et al. 2016), varias especies de mamíferos dispersan las semillas de forma efectiva (I. M. Barberis, observación personal).

\section{Procedimiento de muestreo}

En abril de 2011 se seleccionaron tres sitios de muestreo en los sectores elevados, separados entre sí por una matriz de herbáceas sin cobertura leñosa, a una distancia de al menos $500 \mathrm{~m}$. Dado el comportamiento territorial y el tamaño reducido de los territorios de los principales polinizadores de $B$. serra (picaflores e insectos), esta distancia podría considerarse suficientemente amplia como para que el flujo de polen dentro de los sitios sea mayor que entre los sitios (J. L. Vesprini, observación personal). Por el contrario, esta distancia no es suficiente para asegurar que las semillas provenientes de los frutos consumidos por algunos mamíferos (e.g., zorro gris, zorro de monte, aguará popé) no sean dispersadas entre sitios. En cada sitio se seleccionaron cuatro parches o colonias y en cada uno se seleccionaron al azar cuatro plantas en estado reproductivo que estuvieran en buenas condiciones y provinieran de reproducción asexual (total: 48 plantas). Se considera que las plantas de un mismo parche se habrían originado de unos pocos genotipos mediante reproducción asexual; para asegurarse de ello se eligieron aquellas plantas que estuvieran vinculadas por estolones. Para caracterizar el ambiente lumínico se tomó una fotografía hemisférica del dosel por encima de cada planta; las fotografías fueron tomadas al atardecer, en dos días consecutivos, tratando de que la diferencia de tiempo entre la primera y la última fotografía del día fuera lo menor posible. Luego, estas imágenes se analizaron con el software Gap Light Analyzer, versión 2.0) (Frazer et al. 1999). A cada planta se le midió la altura, el diámetro, el largo y el ancho de las hojas. Además, se cosechó la infrutescencia de cada planta, se midió el perímetro y se contó el número de frutos. Se seleccionaron cinco frutos maduros al azar, y se midió el largo y ancho. Los frutos se abrieron con un bisturí y se contó el número de semillas. Por último, los frutos se secaron en estufa a $80{ }^{\circ} \mathrm{C}$ hasta peso constante y se pesaron en una balanza de precisión (Scaltec SBA32; máx=120 g; d=0.0001 g; Goettingen, Alemania).

\section{Análisis de datos}

Para determinar el grado de variación de las variables vegetativas y reproductivas se utilizó el coeficiente de variación (Crawley 2013). La escala espacial de variación de los caracteres vegetativos y reproductivos se analizó particionando la varianza de estas variables por medio de modelos jerárquicos anidados (Crawley 2013). Para cada variable se estimaron los efectos aleatorios del sitio y del parche anidado en el sitio mediante la función lmer del paquete lme4 de R (The R Core Team 2018).

Para saber si existían diferencias significativas en la apertura del dosel entre los tres sitios se realizó un análisis de la varianza mediante la función $\mathrm{lm}$ del paquete stats de R (The R Core Team 2018), usando la 'apertura del dosel' como variable respuesta y los 'Sitios' como variable explicativa. Para evaluar el efecto de la luz y del tamaño de la planta sobre el tamaño de la infrutescencia, a través del número y tamaño de los frutos, se realizó un modelo de ecuaciones estructurales (Figura 2a) utilizando la función psem del paquete piecewiseSEM (Lefcheck 2016). Para evitar la colinealidad entre las variables medidas y seleccionar aquellas a utilizar en el modelo se llevó a cabo un análisis de componentes principales (PCA) con los caracteres vegetativos y reproductivos mediante la función prcomp del paquete stats de R (The R Core Team 2018). Como resultado, para describir el tamaño de la planta se seleccionó la medición de su diámetro y para el tamaño del fruto, el ancho de los frutos. Las variables 'número de frutos' y 'número de semillas' fueron transformadas mediante log10. El modelo de ecuaciones estructurales se generó incorporando modelos lineales generales mixtos (función lmer) considerando la estructura jerárquica planteada anteriormente. Para aquellos modelos que contenían dos o más variables explicativas se evaluó la importancia de la interacción entre ambas mediante comparación de modelos que incluían o no la interacción (Crawley 2013). Además, para cada uno se realizó un análisis de la varianza con suma de cuadrados tipo III. El ajuste del modelo fue evaluado usando la prueba d-separation de Shipley con el estadístico C de Fisher (Lefcheck 2016). Los efectos indirectos de la luz sobre el diámetro de la infrutescencia fueron estimados como la sumatoria del producto de los coeficientes estandarizados de las variables intermedias para cada una de las vías alternativas (Pugesek et al. 2003). Los resultados se expresan como 
a)

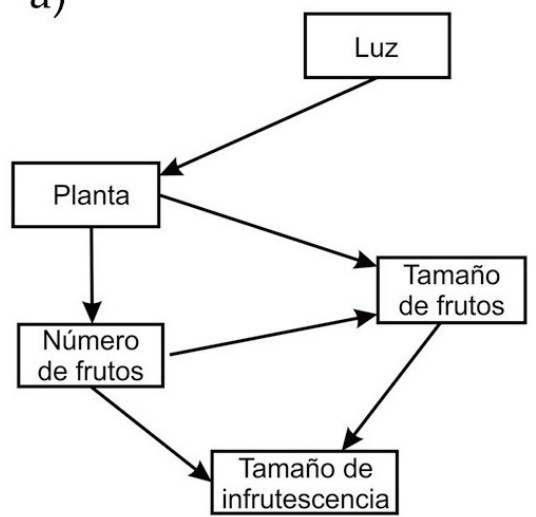

b)

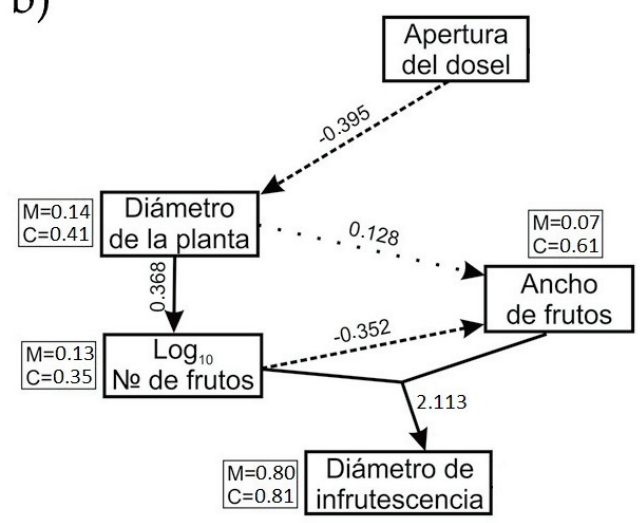

Figura 2. Modelos del diagrama de paso. a) Modelo propuesto para explicar los efectos del ambiente lumínico sobre el diámetro de la infrutescencia de Bromelia serra. Las flechas representan las relaciones teóricas entre las variables incluidas en el modelo. b) Resultados del modelo de diagrama de paso. Para cada flecha se muestran los coeficientes de paso estandarizados (i.e., efectos directos). Las flechas continuas indican efectos positivos, las segmentadas indican efectos negativos y la flecha punteada indica un efecto no significativo. Para las variables dependientes se especifican los valores de $\mathrm{R}^{2}$ marginal (M) (i.e., proporción de la varianza explicada por factores fijos) y $\mathrm{R}^{2}$ condicional (C) (i.e., proporción de la varianza explicada por factores fijos y factores aleatorios). Figure 2. a) Proposed path diagram model for explaining the effects of light environment on infructescence diameter of Bromelia serra. Arrows represent the theoretical relationships between the variables included in the model. b) Results of the path diagram. For each arrow, the standardized path coefficients (i.e., direct effects) are shown. Solid arrows denote positive effects, segmented arrows denote negative effects, and the dotted arrow denote a non-significant effect. For each dependent variable, marginal $\mathrm{R}^{2}$ values $(\mathrm{M})$ (i.e., proportion of variance explained by fixed factors) and conditional $\mathrm{R}^{2}$ values (C) (i.e., proportion of variance explained by fixed and random factors) are shown.

medias \pm un desvío estándar; también se incluyen los coeficientes de variación $(\mathrm{CV})$ y el estadístico $F$ de Snedecor (F) en las pruebas estadísticas que corresponda.

\section{Resultados}

\section{Caracterización general}

Las variables que presentaron mayor variabilidad entre las plantas de $B$. serra fueron el número de frutos por infrutescencia (29.16 $\pm 14.77 ; C V=50.65)$ y el número de semillas por fruto $(23.62 \pm 11.19 ; \mathrm{CV}=47.37)$, seguidas por las variables reproductivas relacionadas con el peso del fruto $(1.57 \pm 0.54$ g; $C V=34.09)$ y el diámetro de la infrutescencia $(9.24 \pm 1.77 \mathrm{~cm} ; C V=19.17)$, y las variables vegetativas relacionadas con el tamaño de la planta, como la altura $(0.59 \pm 0.18 \mathrm{~m} ; \mathrm{CV}=29.59)$, el diámetro $(1.18 \pm 0.22 \mathrm{~m} ; \mathrm{CV}=18.84)$ y el largo de la hoja más larga $(0.75 \pm 0.14 \mathrm{~m} ; \mathrm{CV}=18.86)$. Por el contrario, las variables reproductivas relacionadas con el tamaño del fruto, como el largo del fruto $(3.31 \pm 0.42 \mathrm{~cm} ; C V=12.65)$ y el ancho del fruto $(2.05 \pm 0.26 \mathrm{~cm} ; C V=12.46)$, presentaron baja variación entre las plantas medidas.

Las variables difirieron de forma marcada en la fracción de la varianza asociada a su disposición espacial (i.e., factores aleatorios sitios y parches dentro de los sitios) (Figura 3). Más del $40 \%$ de la varianza de las variables vegetativas (e.g., altura, largo y ancho de la hoja más larga) y del número de semillas fue capturada por factores aleatorios. En cambio, para el resto de las variables reproductivas (i.e., peso, ancho y largo de fruto, número de frutos y diámetro de la infrutescencia) y el diámetro de la planta, la varianza capturada por los factores aleatorios fue menor al 30\% (Figura 3). Se registraron también diferencias en la cantidad de varianza explicada por cada nivel jerárquico. El nivel de parche fue más relevante para las variables vegetativas y el número de semillas que para las variables reproductivas (Figura 3), mientras que el nivel de sitio lo fue para la altura, el diámetro de la infrutescencia, el peso y el número de frutos (Figura 3).

Los dos primeros componentes del análisis de componentes principales explicaron un $68 \%$ de la variación total de la matriz planta $\times$ variables (Figura 4). El primer eje representó al tamaño general de las plantas, y mostró una asociación positiva con la altura, el diámetro, el largo y ancho de las hojas, el tamaño de las infrutescencias y el número, tamaño y peso de los frutos y el número de semillas. En cambio, el segundo eje contrastó a las plantas con valores relativos mayores de sus variables reproductivas (asociación 


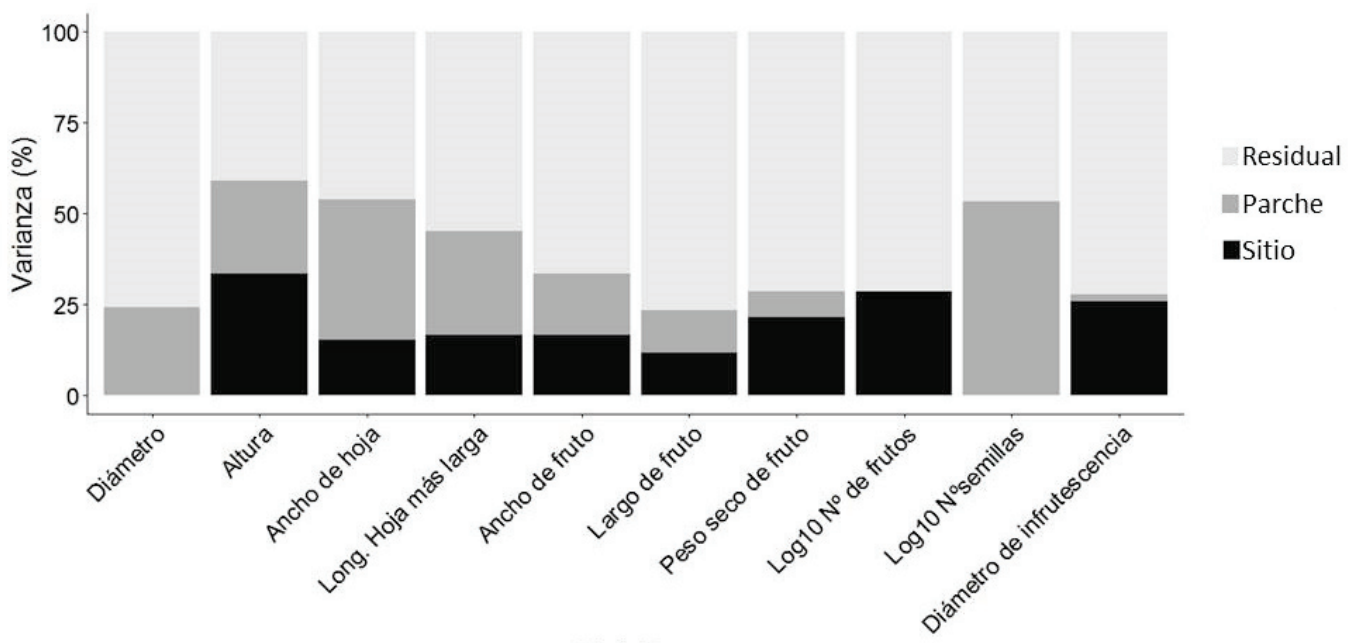

Variables

Figura 3. Fracción de la varianza capturada por estructuración espacial (factores aleatorios Sitio y Parche dentro de Sitio) para las variables vegetativas y reproductivas medidas en plantas de Bromelia serra del quebrachal. Figure 3. Fraction of the variance captured by the spatial distribution (random factors Sites and Patches within Sites) for vegetative and reproductive variables of the Bromelia serra plants in the quebrachal.

positiva con el tamaño y el peso de los frutos y el número de semillas) con aquellas que poseían mayores valores relativos de sus variables vegetativas y el tamaño de las infrutescencias (Figura 4).

\section{Efectos del ambiente lumínico}

No se registraron diferencias significativas en la apertura del canopeo entre los sitios (A: $40.6 \pm 8.66 \%$; B: $39.7 \pm 11.13 \%$; C: $38.9 \pm 6.99 \%$;
$\left.\mathrm{F}_{2,47}=0.136, P=0.874\right)$. El modelo de ecuaciones estructurales propuesto para explicar el tamaño de las infrutescencias (Fisher $\mathrm{C}=10.703$; g.l. $=8 ; P=0.219$ ) (Figura 2b) demuestra que las plantas bajo un dosel más abierto presentan menor tamaño ( $\mathrm{F}=10.517$; g.1. $=1 ; P=0.002)$ (Figura 5a), y, en consecuencia, presentan un menor número de frutos $(\mathrm{F}=9.223$; g.l. $=1$; $P=0.004$ ) (Figura $5 b$ ). Según este modelo, el tamaño de los frutos está determinado por el número de los frutos ( $\mathrm{F}=6.727 ; \mathrm{g} .1 .=1 ; P=0.013)$,

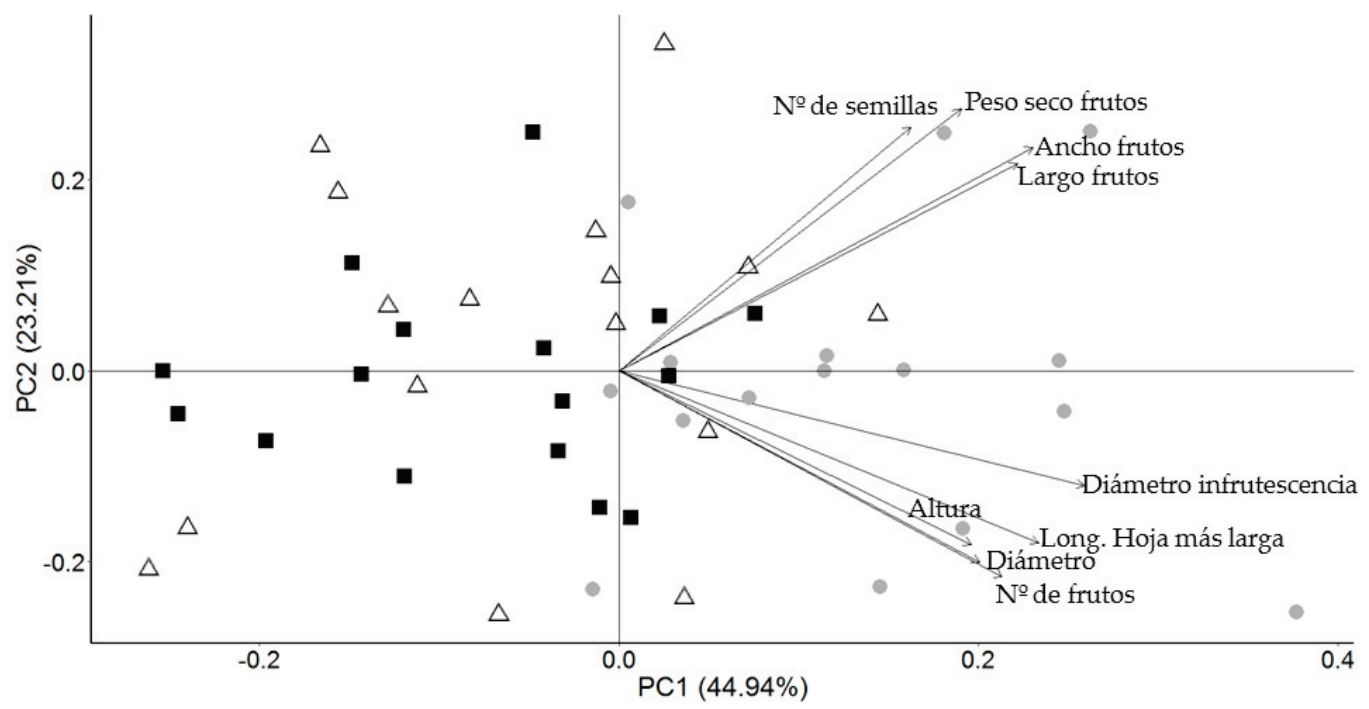

Figura 4. Diagrama de dispersión de los dos primeros ejes del análisis de componentes principales para los caracteres vegetativos y reproductivos de Bromelia serra. Los círculos representan las plantas del sitio A, los triángulos las del sitio B y los cuadrados las del sitio C. Se muestra el porcentaje de variación explicado por cada eje. Figure 4. Dispersal plot for the first two axes of the Principal Component Analysis for vegetative and reproductive traits of Bromelia serra. The circles denote plants from site A, the triangles those from site B and the squares those from site $\mathrm{C}$. The percentage of variance explained by each axis is shown. 


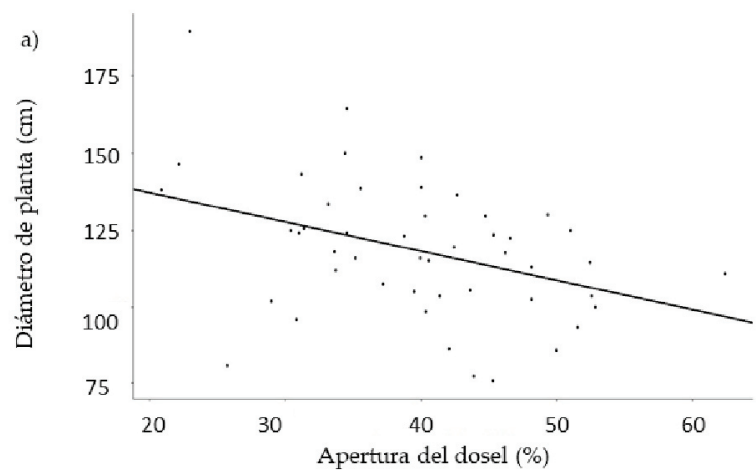

b)
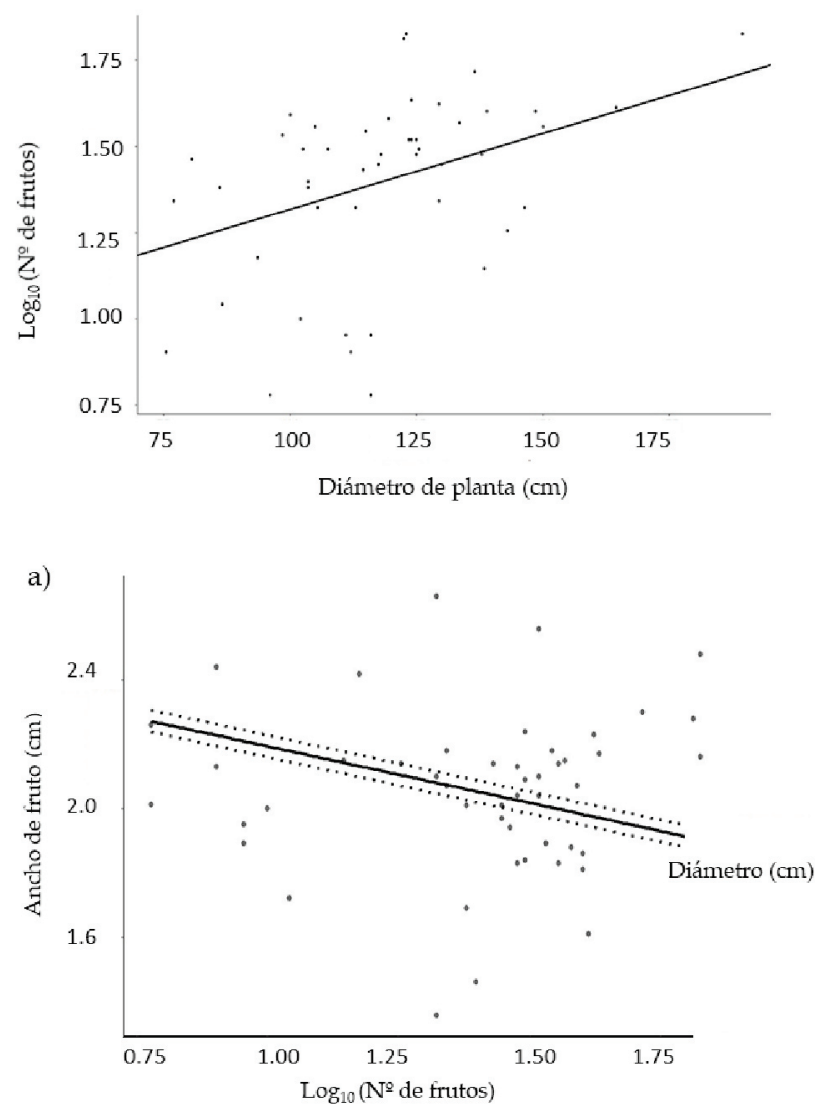

b)

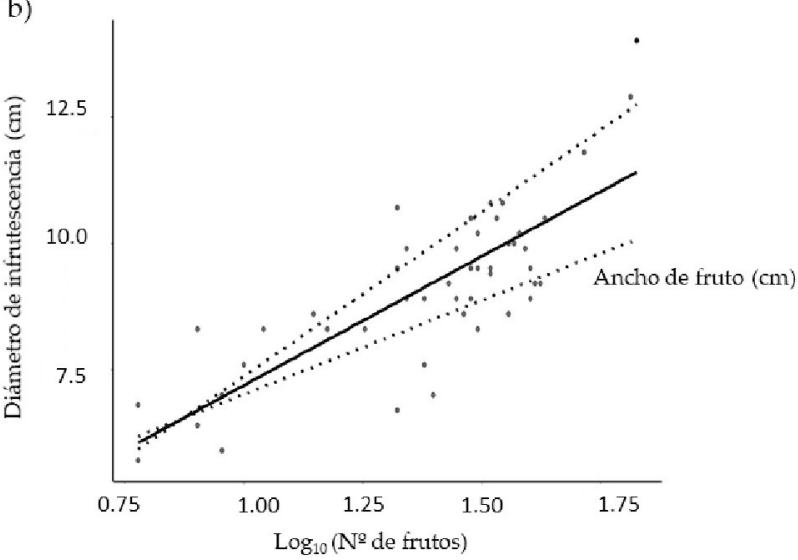

Figura 5.Modelo de ecuaciones estructurales de las variables reproductivas. a) Variación del diámetro de la planta en función de la apertura del dosel $\left(\mathrm{R}^{2}=0.39\right)$. b) Variación del número de frutos en función del diámetro de la planta $\left(\mathrm{R}^{2}=0.35\right)$. Figure 5. a) Plant diameter variation in relation to canopy openness $\left(\mathrm{R}^{2}=0.39\right)$. b) Fruit number variation in relation to plant diameter $\left(\mathrm{R}^{2}=0.35\right)$.

Figura 6. Modelo de ecuaciones estructurales de las variables reproductivas. a) Variación del ancho del fruto en función del número de frutos por infrutescencia $\left(\log _{10} \mathrm{~N}^{\mathrm{o}}\right.$ de frutos) y del diámetro de la planta (diámetro) $\left(\mathrm{R}^{2}=0.61\right)$. b) Variación del diámetro de la infrutescencia en función del número de frutos ( $\log _{10} \mathrm{~N}^{\mathrm{o}}$ de frutos) $\mathrm{y}$ del ancho del fruto $\left(\mathrm{R}^{2}=0.81\right)$. Las líneas sólidas indican los estimadores centrales y las punteadas los desvíos estándar. Figure 6. a) Fruit width variation in relation to the number of fruits per infructescence ( $\log _{10}$ number of fruits) and to the plant diameter. b) Variation of the infructescence diameter in relation to fruit number $\left(\log _{10}\right.$ number of fruits), and fruit width. Solid lines indicate the central estimates and the dotted lines the standard deviance. 


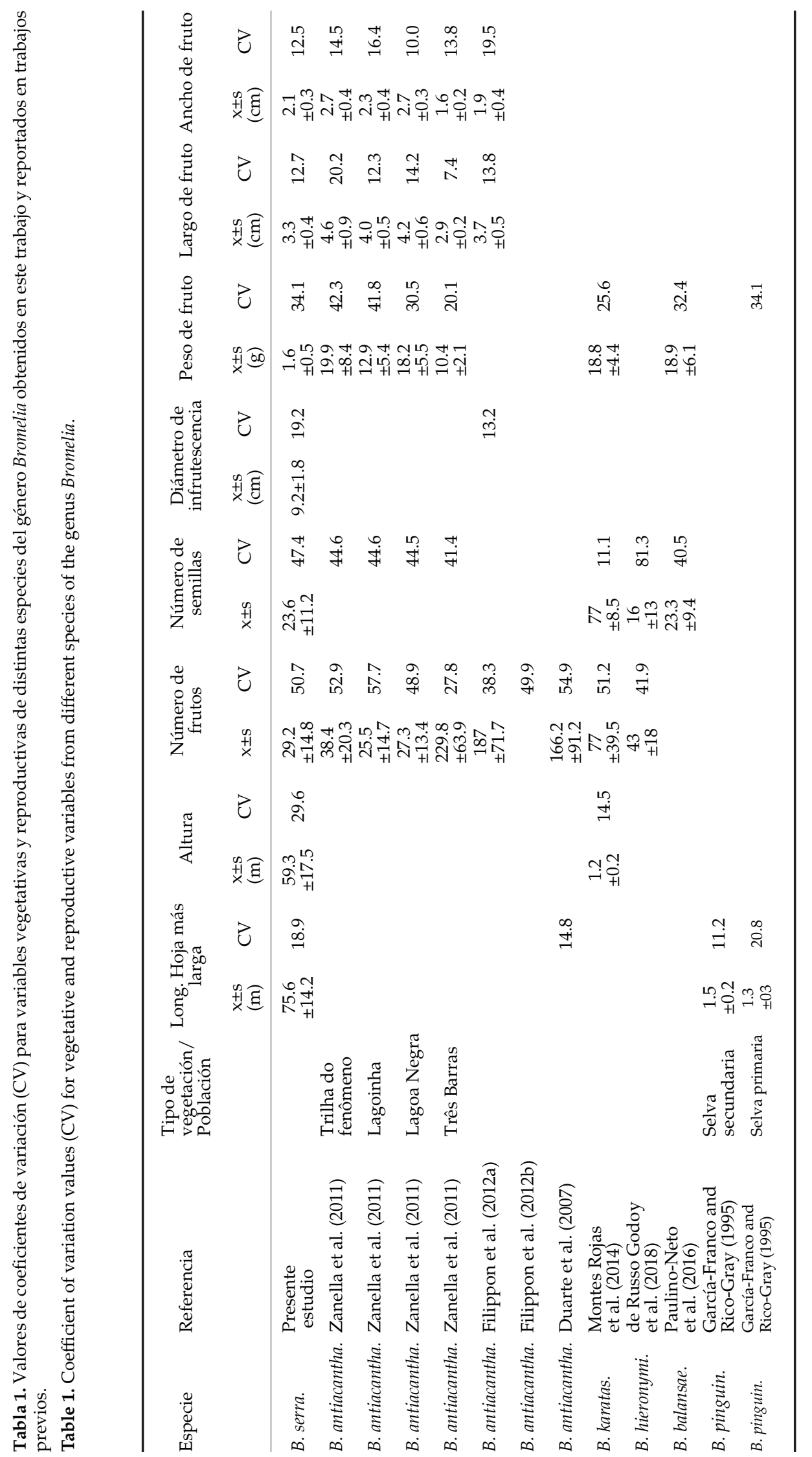


pero no por el tamaño de la planta $(\mathrm{F}=0.962$; g.l.=1; $P=0.332)$; a mayor número de frutos, menor es el tamaño de los mismos (Figuras 2 b y 6a). El tamaño de la infrutescencia está determinado por la interacción entre el número y el tamaño de los frutos $(\mathrm{F}=6.191$; g.1.=1; $P=0.017$ ) (Figuras $2 \mathrm{~b}$ y 6b); cuando el número de frutos es elevado, el diámetro de la infrutescencia aumenta con el tamaño de los frutos, mientras que cuando el número de frutos es bajo, la infrutescencia no varía con el tamaño de los mismos (Figura 6b). En consecuencia, la apertura del dosel influyó de forma negativa, aunque con baja magnitud, sobre el diámetro de la infrutescencia debido a un efecto indirecto mediado por el tamaño de la planta y el número y tamaño de los frutos (efecto indirecto=-0.199) (Figura 2b).

\section{DiscUSIÓN}

\section{Caracterización general}

Los valores de coeficientes de variación $(\mathrm{CV})$ de las variables vegetativas y reproductivas de los individuos de $B$. serra son similares a los valores reportados para otras especies del género Bromelia, en las que la mayor variación se registró en el número de frutos por planta y en el número de semillas por fruto, y las menores variaciones en el tamaño de los frutos (Tabla 1). Debe tenerse en cuenta que en nuestro estudio sólo se midieron cinco frutos de cada infrutescencia, por lo que la variabilidad en el número de semillas por fruto podría haber disminuido si se hubiesen medido más frutos. A su vez, para Bromelia hieronymi Mez se reportaron plantas que producen algunos frutos partenocárpicos (de Russo Godoy et al. 2018). Si bien en nuestro estudio no registramos frutos sin semillas, no podemos descartar la posibilidad de partenocarpia en B. serra. Por otro lado, la variabilidad en el tamaño de los frutos, medidos en ancho, largo y peso, podría estar sesgada por el número y la posición que ocupaba cada fruto, ya que se conoce que el tamaño de los frutos puede variar según la posición dentro de la infrutescencia (Li et al. 2010).

La variabilidad entre plantas de B. serra debida a su distribución espacial (porcentaje de varianza explicado por factores aleatorios) fue mayor para las variables vegetativas que para las variables reproductivas. Para las primeras, la mayor proporción de esta varianza se registró a nivel de parche, mientras que para las variables reproductivas, la mayor variabilidad fue entre sitios. Esto parecería ser contraintuitivo, ya que se espera que a nivel de parche las plantas sean similares entre sí, dado que se multiplican principalmente mediante reproducción asexual, y que entre parches haya mayores diferencias entre plantas ya que la colonización de los distintos parches y sitios se realiza mediante reproducción sexual. Sin embargo, dadas la heterogeneidad ambiental de estos bosques y la plasticidad de esta especie (Barberis et al. 2014, 2017), los resultados observados podrían atribuirse a una mayor plasticidad fenotípica de los caracteres vegetativos que de los reproductivos, tal como fuera sugerido para otras especies de bromeliáceas (Freire et al. 2018). Para comprobar esto se requieren estudios que midan la variabilidad de caracteres vegetativos y reproductivos entre plantas originadas por reproducción sexual y asexual dentro y entre parches. Bromelia serra es un buen modelo para este tipo de estudios, ya que dentro de un parche se pueden distinguir las rosetas provenientes de cada forma de reproducción debido a que sus estolones subterráneos perduran durante mucho tiempo.

\section{Efectos del ambiente lumínico}

Lasplantas de B. serrabajodoselesmásabiertos presentaron un tamaño menor que las plantas que crecieron a la sombra. Patrones similares de plasticidad fenotípica se observaron en numerosas especies de bromeliáceas terrestres (Lee et al. 1989; Cavallero et al. 2009; Barberis et al. 2017; Montero et al. 2017). Estos cambios estructurales en plantas creciendo en distintos hábitats lumínicos se relacionaron con adaptaciones para la captura de luz, como hojas más largas y angostas en plantas establecidas en hábitats más sombreados, y más cortas, anchas y erectas en hábitats más luminosos (Cogliatti-Carvalho et al. 1998; Lenzi et al. 2006; Cavallero et al. 2009; Barberis et al. 2017). Las diferencias entre hábitats en la arquitectura, la morfología y la anatomía de las hojas resultan de un compromiso entre el área fotosintética (para la captura de luz) y el soporte mecánico (Cavallero et al. 2011).

En el presente estudio observamos que el tamaño de la infrutescencia de $B$. serra está asociado positivamente con el tamaño de la planta, tal como lo reportaran Montero et al. (2017). Así, un aumento en el tamaño de las plantas suele traducirse en un aumento del esfuerzo reproductivo (Mantovani and Iglesias 2009), con mayor cantidad de biomasa 
asignada a estructuras reproductivas (Weiner 2004). Un patrón similar se reportó para $A$ comosus y para B. antiacantha: las plantas con hojas más grandes presentaron inflorescencias más grandes que las plantas más pequeñas (Bartholomew et al. 2003; Duarte et al. 2007). No obstante, se debe mencionar que la relación entre el peso de la planta y el peso de la infrutescencia es compleja, ya que depende de las condiciones ambientales durante la formación y del crecimiento de los frutos (Bartholomew et al. 2003).

Otra observación en coincidencia con Montero et al. (2017) es que el ambiente lumínico tuvo un efecto indirecto sobre la reproducción: las plantas de $B$. serra ubicadas en áreas más expuestas fueron más pequeñas y, por consiguiente, tuvieron infrutescencias más pequeñas. Un patrón similar se observó en $A$. comosus, que tuvo menor rendimiento, menor tamaño de fruto promedio y mayor porcentaje de frutos quemados por el sol en monocultivos que cuando se realizó cultivo intercalado con mandioca, que le hizo sombra (Custódio et al. 2016). Para otras especies existen patrones diferentes según el área de estudio. En el caso de $A$. distichantha creciendo en quebrachales en nuestro mismo sitio de estudio (Freire et al. 2018) se registró un mayor número de frutos por infrutescencias en plantas bajo sombra, mientras que en la Mata Atlántica brasileña, las plantas expuestas a mayor luz tuvieron mayor número de frutos por infrutescencia y mayor número de semillas por frutos (Scrok and Varassin 2011). Estas diferencias entre regiones podrían atribuirse a otros factores bióticos como el tipo de polinizador y la frecuencia de visita. Por ejemplo, Kilkenny y Galloway (2008) reportan que las plantas al sol recibieron más visitas de polinizadores que las plantas ubicadas en la sombra, aunque aclaran que este patrón depende de la especie de polinizador y que sus comportamientos pueden variar con las condiciones de cada región de estudio.

Para A. comosus, el tamaño de la infrutescencia está condicionado por el número y el tamaño de los frutos (Bartholomew et al. 2003; Li et al. 2010). Además, los frutos de esta especie ubicados en la base de la infrutescencia son de menor tamaño que los ubicados en el ápice (Li et al. 2010). Para B. serra, el modelo de ecuaciones estructurales indicó que el tamaño de la infrutescencia está determinado por la interacción entre el número de frutos y el tamaño de los mismos. En nuestro estudio, los frutos medidos fueron seleccionados al azar, lo que seguramente aumentó la relevancia del error muestral al comparar entre plantas. En futuros estudios se debería tener en cuenta la ubicación de los frutos dentro de las infrutescencias para estratificar el muestreo.

\section{Consideraciones finales}

Este estudio se centró exclusivamente en describir la variabilidad asociada a la disposición espacial de $B$. serra en este ambiente y evaluar el efecto del ambiente lumínico sobre sus estructuras reproductivas sexuales como el número de frutos y semillas. Teniendo en cuenta que $B$. serra se reproduce tanto sexual como asexualmente (Smith and Downs 1979; Bianchi et al. 2000; Klekailo et al. 2012), entonces la asignación de recursos a estructuras reproductivas sexuales podría estar condicionada por la producción de hijuelos relacionada con la clonalidad (Silva and Varassin 2016). Sería conveniente llevar a cabo estudios que consideren el efecto del ambiente lumínico sobre la asignación individual/poblacional de recursos a la reproducción sexual y asexual teniendo en cuenta el tamaño de las plantas (Weiner 2004).

Si bien varios de los patrones descriptos en este trabajo parecen sólidos y compatibles con resultados de trabajos previos, los resultados podrían mejorarse con muestreos que a) incluyeran mayor cantidad de sitios, lo suficientemente distanciados entre sí para asegurar que no haya intercambio genético entre sitios y que tuvieran diferente apertura del canopeo para poder comparar entre ellos, b) midieran mayor cantidad de plantas por parche, lo que permitiría aumentar el número total de muestras, c) midieran todos los frutos de cada infrutescencia o al menos se tuviera en cuenta sus posiciones relativas dentro de las mismas, y d) seleccionaran, en un mismo parche, plantas provenientes de reproducción sexual y asexual para evaluar la plasticidad intra y entre individuos.

Agradecimientos. Agradecemos al Ministerio de la Producción de la Provincia de Santa Fe por permitirnos trabajar en Las Gamas. Agradecemos a F. Hernández y B. Rigalli por su ayuda durante el trabajo de campo. Este estudio fue financiado por la Agencia Nacional de Promoción Científica y Tecnológica (PICT2010-1614) y por la Universidad Nacional de Rosario (AGR-290). Los comentarios y sugerencias del editor y los revisores ayudaron a mejorar notablemente la calidad de este trabajo. 


\section{REFERENCIAS}

Amat, A. G. 1988. Identificación de las especies argentinas del género Bromelia L. (Bromeliaceae: Bromelioideae) mediante caracteres espermatológicos. Acta Farmacéutica Bonaerense 7:25-32.

Arenas, P., and S. C. Arroyo. 1988. Las especies comestibles del género Bromelia (Bromeliaceae) del Gran Chaco. Candollea 43:645-660.

Barbará, T., C. Lexer, G. Martinelli, S. Mayo, M. F. Fay, and M. Heuertz. 2008. Within-population spatial genetic structure in four naturally fragmented species of a neotropical inselberg radiation, Alcantarea imperialis, A. geniculata, A. glaziouana and A. regina (Bromeliaceae). Heredity 101:285-296. https:// doi.org/10.1038/hdy.2008.65.

Barberis, I. M., and J. P. Lewis. 2005. Heterogeneity of terrestrial bromeliad colonies and regeneration of Acacia praecox (Fabaceae) in a humid-subtropical-Chaco forest. Argentina. Revista de Biología Tropical 53:377-385. https: / /doi.org/ 10.15517/rbt.v53i3-4.14600.

Barberis, I. M., E. F. Pire, and J. P. Lewis. 1998. Spatial heterogeneity and woody species distribution in a Schinopsis balansae (Anacardiaceae) forest of the Southern Chaco. Argentina. Revista de Biología Tropical 46:515-524.

Barberis, I. M., W. B. Batista, E. F. Pire, J. P. Lewis, and R. J. C. León. 2002. Woody population distribution and environmental heterogeneity in a Chaco forest. Argentina. Journal of Vegetation Science 13:607-614. https: / /doi.org/ 10.1111/j.1654-1103.2002.tb02088.x.

Barberis, I. M., P. S. Torres, W. B. Batista, G. Magra, L. Galetti, and J. P. Lewis. 2014. Two bromeliad species with contrasting functional traits partition the understory space in a Southamerican xerophytic forest: correlative evidence of environmental control and limited dispersal. Plant Ecology 215:143-153.

Barberis, I. M., J. M. Cárcamo, J. I. Cárcamo, and J. Albertengo. 2017. Phenotypic plasticity in Bromelia serra Griseb.: morphological variations due to plant size and habitats with contrasting light availability. Revista Brasileira de Biociências 15:143-150.

Bartholomew, D. P., E. Malézieux, G. M. Sanewski, and E. Sinclair. 2003. Inflorescence and fruit development and yield. Pp. 167-202 en D. P. Bartholomew, R. E. Paull and K. G. Rohrbach (eds.). The pineapple: botany, production, and uses. CABI, Wallingford, UK. https://doi.org/10.1079/9780851995038.0167.

Bazzaz, F. A. 1991. Habitat selection in plants. The American Naturalist 137:S116-S130. https://doi.org/10.1086/ 285142.

Bazzaz, F. A., D. D. Ackerly, and E. G. Reeki. 2000. Reproductive allocation in plants. Pp 1-29 en M. Fenner (ed.). Seeds: the ecology of regeneration in plant communities. CABI, Wallingford, UK. https://doi.org/10.1079/ 9780851994321.0001.

Benzing, D. H. 2000. Bromeliaceae. Profile of an adaptive radiation. Cambridge University Press, Cambridge, UK. https://doi.org/10.1017/CBO9780511565175.

Bianchi, M. B., P. E. Gibbs, D. E. Prado, and J. L. Vesprini. 2000. Studies on the breeding systems of understorey species of a Chaco woodland in NE Argentina. Flora 195:339-348. https:/ /doi.org/10.1016/S0367-2530(17)30992-1.

Caffini, N. O., C. L. Natalucci, N. S. Priolo, and M. S. Buttazzoni. 1988. Proteasas de Bromeliaceae. IV. Aislamiento de una fitoproteasa sulfhidrílica presente en frutos de Bromelia serra Griseb. Acta Farmacéutica Bonaerense 7:9-14.

Canela, M., and M. Sazima. 2005. The pollination of Bromelia antiacantha (Bromeliaceae) in Southeastern Brazil: ornithophilous versus melittophilous features. Plant Biology 7:411-416. https://doi.org/10.1055/s-2005-865619.

Cavallero, L., D. López, and I. M. Barberis. 2009. Morphological variation of Aechmea distichantha (Bromeliaceae) in a Chaco forest: habitat and size-related effects. Plant Biology 11:379-391. https://doi.org/10.1111/j.14388677.2008.00123.x.

Cavallero, L., L. Galetti, D. López, J. McCargo, and I. M. Barberis. 2011. Morphological variation of the leaves of Aechmea distichantha Lem. plants from contrasting habitats of a Chaco forest: a trade-off between leaf area and mechanical support. Revista Brasileira de Biociências 9:455-464.

Cogliatti-Carvalho, L., D. R. Almeida, and C. F. D. Rocha. 1998. Phenotypic response of Neoregelia johannis (Bromeliaceae) dependent on light intensity reaching the plant microhabitat. Selbyana 19:240-244.

Crawley, M. J. 2013. The R Book. John Wiley and Sons, Chichester, UK.

Custódio, R. A., S. E. A. Neto, P. C. P. F. Junior, R. C. A. Neto, and I. F. Silva. 2016. Morpho-anatomy of leaves and yield of pineapple plant in intercropping with cassava. Bioscience Journal 32:839-848. https:/ / doi.org/10.14393/BJv32n4a2016-26827.

Duarte, A. S., C. Vieira da Silva, A. Puchalski, M. Mantovani, J. S. Silva, and M. S. Reis. 2007. Estrutura demográfica e produção de frutos de Bromelia antiacantha Bertol. Revista Brasileira de Plantas Medicinais. Botucatu 9:106-112.

Fassinou Hotegni, V. N., W. J. M. Lommen, E. K. Agbossou, and P. C. Struik. 2015. Influence of weight and type of planting material on fruit quality and its heterogeneity in pineapple [Ananas comosus (L.) Merrill]. Frontiers in Plant Science 5:798. https://doi.org/10.3389/fpls.2014.00798.

Filippon, S., C. D. Fernandes, D. K. Ferreira, A. S. Duarte, and M. S. Reis. 2012a. Produção de frutos para uso medicinal em Bromelia antiancatha (caraguatá): fundamentos para um extrastivismo sustentável. Revista Brasileira de Plantas Medicinais 14:506-513.

Filippon, S., C. D. Fernandes, D. K. Ferreira, D. L. S. da Silva, G. Altrak, A. S. Duarte, and M.S. Reis. 2012b. Bromelia antiacantha Bertol. (Bromeliaceae): caracterização demográfica e potencial de manejo em uma população no Planalto Norte Catarinense. Biodiversidade Brasileira 2: 83-91. https:/ / doi.org/10.1590/S1516-05722012000300013.

Frazer, G. W., C. D. Canham, and K. P. Lertzman. 1999. Gap Light Analyzer (GLA). Simon Fraser University and The Institute of Ecosystem Studies. Burnaby, British Columbia and Millbrook, New York. 
Freire, R. M., I. M. Barberis, and J. L. Vesprini. 2018. Reproductive traits and floral visitors of Aechmea distichantha plants growing in different habitats of a South American xerophytic forest. Rodriguésia 69:385-396. https://doi.org/ 10.1590/2175-7860201869210.

García-Franco, J. G., and V. Rico-Gray. 1995. Population structure and clonal growth in Bromelia pinguin L. (Bromeliaceae) in dry forests of coastal Veracruz, Mexico. Tulane Studies in Zoology and Botany 30:27-37.

González-Salvatierra, C., J. L. Andrade, R. Orellana, L. M. Peña-Rodríguez, and C. Reyes-García. 2013. Microambiente lumínico y morfología y fisiología foliar de Bromelia karatas (Bromeliaceae) en una selva baja caducifolia de Yucatán. México. Botanical Sciences 91:75-84. https://doi.org/10.17129/botsci.403.

Grime, J. P., and S. Pierce. 2012. The evolutionary strategies that shape ecosystems. John Wiley and Sons, Oxford, UK. https://doi.org/10.1002/9781118223246.

Harper, J. L. 1977. Population biology of plants. Academic Press, Londres, UK.

Hendrix, S. D. 1988. Herbivory and its impact on plant reproduction. Pp. 246-263 en J. Lovett Doust, and L. Lovett Doust (eds.). Plant reproductive ecology: patterns and strategies. Oxford University Press, Oxford, UK.

Herrera, C. M. 2017. The ecology of subindividual variability in plants: patterns, processes, and prospects. Web Ecology 17:51-64. https://doi.org/10.5194/we-17-51-2017.

Keddy, P. A. 2017. Plant ecology: origins, processes, consequences. Cambridge University Press, Cambridge, UK.

Kilkenny, F. F., and L. F. Galloway. 2008. Reproductive success in varying light environments: direct and indirect effects of light on plants and pollinators. Oecologia 155:247-255. https:/ / doi.org/10.1007/s00442-007-0903-z.

Klekailo, G. N., D. H. Tuesca, and I. M. Barberis. 2012. Efectos de la temperatura, el ambiente lumínico y la escarificación sobre la germinación de semillas de Bromelia serra Griseb (Bromeliaceae). Revista Brasileira de Sementes 34:605-612. https://doi.org/10.1590/S0101-31222012000400011.

Lee, H. S. J., U. Lüttge, E. Medina, J. A. C. Smith, W. J. Cram, M. Díaz, H. Griffiths, M. Popp, C. Schäfer, K.-H. Stimmel, and B. Thonke. 1989. Ecophysiology of xerophytic and halophytic vegetation of a coastal alluvial plain in northern Venezuela. III. Bromelia humilis Jacq., a terrestrial CAM bromeliad. New Phytologist 111:253-271. https://doi.org/ 10.1111/j.1469-8137.1989.tb00690.x.

Lefcheck, J. S. 2016. piecewiseSEM: Piecewise structural equation modelling in R for ecology, evolution, and systematics. Methods in Ecology and Evolution 7:573-579. https://doi.org/10.1111/2041-210X.12512.

Lenzi, M., J. Z. Matos, and A. I. Orth. 2006. Variação morfológica e reprodutiva de Aechmea lindenii (E. Morren) Baker var. lindenii (Bromeliaceae). Acta Botanica Brasilica 20:487-500. https://doi.org/10.1590/S0102-33062006000200024.

Lewis, J. P. 1991. Three levels of floristical variation in the forests of Chaco. Journal of Vegetation Science 2:125-130. https://doi.org/10.2307/3235905.

Lewis, J. P., E. F. Pire, and I. M. Barberis. 1997. Structure, physiognomy and floristic composition of a Schinopsis balansae (Anacardiaceae) forest in the Southern Chaco, Argentina. Revista de Biología Tropical 45:1013-1020c.

Li, Y.-H., Z. Zhang, and G.-M. Sun. 2010. Changes in cell number and cell size during pineapple (Ananas comosus L.) fruit development and their relationship with fruit size. Australian Journal of Botany 58:673-678. https://doi.org/ $10.1071 /$ BT10225.

Liu, F., Y-Y. Liao, L. Wei, J.-M. Chen, Q.-F. Wang, and T. J. Motley. 2010. The effect of pollination on resource allocation among sexual reproduction, clonal reproduction, and vegetative growth in Sagittaria potamogetifolia (Alismataceae). Ecological Research 25:495-499. https://doi.org/10.1007/s11284-009-0679-1.

Lovett Doust, J., and L. Lovett Doust. 1988. Plant reproductive ecology: patterns and strategies. Oxford University Press, Ithaca, USA.

Mantovani, A., and R. R. Iglesias. 2009. Size-dependent allocation of biomass to ancillary versus flowers of the inflorescences of the epiphyte Tillandsia stricta Soland (Bromeliaceae). Acta Botanica Brasilica 23:130-135. https: //doi.org/10.1590/S0102-33062009000100016.

Montero, G., G. Klekailo, R. Freire, P. Torres, A. Cococcioni, and I. Barberis. 2017. Infructescence size has a larger effect than light environment on the abundance of different arthropod feeding guilds dwelling on the infructescences of a terrestrial bromeliad in a xerophytic forest. Studies on Neotropical Fauna and Environment 52:216-227. https: //doi.org/10.1080/01650521.2017.1346044.

Montes Rojas, C., V. F. Terán, R. A. Zuñiga, and Y. E. Caldón. 2014. Descripción morfológica de Bromelia karatas recurso genético promisorio para Patía, Cauca, Colombia. Biotecnología en el Sector Agropecuario y Agroindustrial 12:62-70.

Nájera, M. 1974. Estructuras secretoras de las especies argentinas del género Bromelia (Bromeliaceae). Boletín de la Sociedad Argentina de Botánica 15:384-392.

Overbeek, J. V. 1946. Control of flower formation and fruit size in the pineapple. Botanical Gazette 108:64-73. https: //doi.org/10.1086/335396.

Oyarzabal, M., M. Clavijo, L. J. Oakley, F. Biganzoli, P. Tognetti, I. M. Barberis, H. M. Maturo, R. Aragón, P. I. Campanello, D. E. Prado, M. Oesterheld, and R. J. C. León. 2018. Unidades de vegetación de la Argentina. Ecología Austral 28 : 40-63. https://doi.org/10.25260/EA.18.28.1.0.399.

Parera, A. 2002. Los mamíferos de la Argentina y la región austral de Sudamérica. El Ateneo, Buenos Aires, Argentina.

Paulino-Neto, H., E. Nakano-Oliveira, M. M. de Assis Jardim, and J. Vasconcellos-Neto. 2016. Frugivory in Bromelia balansae (Bromeliaceae): the effect of seed passage through the digestive system of potential seed dispersers on germination in an Atlantic Rainforest, Brazil. Journal of Ecosystem and Ecography 6:2. https://doi.org/10.4172/ 
2157-7625.1000224.

Pugesek, B. H, A. Tomer, and A. von Eye. 2003. Structural equation modeling. Applications in ecological and evolutionary biology. Cambridge University Press, Cambridge, UK. https:/ /doi.org/10.1017/CBO9780511542138.

de Russo Godoy, F. M., M. Lenzi, B. H. dos Santos Ferreira, L. Vicente da Silva, C. Martini Zanella, and G. Matos Paggi. High genetic diversity and moderate genetic structure in the self-incompatible, clonal Bromelia hieronymi (Bromeliaceae). Botanical Journal of the Linnean Society 187:672-688. https:/ / doi.org/10.1093/botlinnean/boy037

Satorre, E. H., R. L. Benech Arnold, G. A. Slafer, E. B. de la Fuente, D. J. Miralles, M. E. Otegui, and R. Savin. 2003. Producción de granos. Bases funcionales para su manejo. Editorial Facultad de Agronomía, Buenos Aires, Argentina.

Scarano, F. R., H. M. Duarte, G. Rôças, S. M. B. Barreto, E. F. Amado, F. Reinert, T. Wendt, A. Mantovani, H. R. P. Lima, and C. F. Barros. 2002. Acclimation or stress symptom? An integrated study of intraspecific variation in the clonal plant Aechmea bromeliifolia, a widespread CAM tank-bromeliad. Botanical Journal of the Linnean Society 140: 391-401. https: / / doi.org/10.1046/j.1095-8339.2002.00112.x.

Scrok, G. J., and I. G. Varassin. 2011. Reproductive biology and pollination of Aechmea distichantha Lem. (Bromeliaceae). Acta Botanica Brasilica 25:571-576. https: / / doi.org/10.1590/S0102-33062011000300009.

Silva, M. A. d. S., and I. G. Varassin. 2016. Effect of rosette size, clonality and spatial distribution on the reproduction of Vriesea carinata (Bromeliaceae) in the Atlantic Forest of Paraná, southern Brazil. Acta Botanica Brasilica 30:401-406. https://doi.org/10.1590/0102-33062016abb0144.

Smith, L. B., and R. J. Downs. 1979. Bromeliaceae. subfamily Bromelioideae. Flora Neotropica Monograph 14:14932142.

The R Core Team. 2018. A language and environment for statistical computing. R version 3.5.1. The R. Foundation for Statistical Computing, Viena, Austria.

Villegas, A. C. 2001. Spatial and temporal variability in clonal reproduction of Aechmea magdalenae, a tropical understorey herb. Biotropica 33:48-59. https://doi.org/10.1111/j.1744-7429.2001.tb00156.x.

Voltolini, C. H., and M. Santos. 2011. Variações na morfo-anatomia foliar de Aechmea lindenii (E. Morren) Baker var. lindenii (Bromeliaceae) sob distintas condições ambientais. Acta Botanica Brasilica 25:2-10. https://doi.org/10.1590/ S0102-33062011000100002.

Waller, D. M. 1988. Plant morphology and reproduction. Pp. 203-227 en J. Lovett Doust, and L. Lovett Doust (ed.). Plant reproductive ecology: patterns and strategies. Oxford University Press, Oxford, UK.

Weiner, J. 2004. Allocation, plasticity and allometry in plants. Perspectives in Plant Ecology. Evolution and Systematics 6:207-215. https://doi.org/10.1078/1433-8319-00083.

Weiner, J., L. G. Campbell, J. Pino, and L. Echarte 2009. The allometry of reproduction within plant populations. Journal of Ecology 97:1220-1233. https:/ /doi.org/10.1111/j.1365-2745.2009.01559.x.

Zanella, C. M., M. Bruxel, G. M. Paggi, M. Goetze, M. V. Buttow, F. W. Cidade, F. Bered. 2011. Genetic structure and phenotypic variation in wild populations of the medicinal tetraploid species Bromelia antiacantha (Bromeliaceae). American Journal of Botany 98:1511-1519. https:/ / doi.org/10.3732/ajb.1000458.

Zimmerman, M. 1988. Nectar production. flowering phenology. and strategies for pollination. Pp. 157-178 en J. Lovett Doust, and L. Lovett Doust (ed.). Plant reproductive ecology: patterns and strategies. Oxford University Press, Oxford, UK. 